\title{
Anchor Pair Selection for Error Correction in Time Difference of Arrival (TDoA) Ultra Wideband (UWB) Positioning Systems
}

\author{
Ben Van Herbruggen, Jaron Fontaine and Eli De Poorter \\ INTEC-IDLab, Ghent University-IMEC, Ghent, Belgium \\ Email: ben.vanherbruggen@ugent.be
}

\begin{abstract}
Ultra wideband positioning systems typically use techniques such as two way ranging (TWR) or time difference of arrival (TDoA) to calculate the position of mobile tags. TDoA techniques require the transmission of only a single packet by the mobile tag, thus providing better scalability, higher update rates and less energy consumption than TWR techniques. However, the TDoA performance degrades heavily when a subset of the anchors are in non-line-of-sight (NLOS) conditions with the tag or with each other. To remedy this, we propose and compare different algorithms to select a subset of anchor pairs before calculating the TDoA position in 3 different conditions: LOS conditions between all devices, NLOS conditions between tag and anchor nodes and NLOS conditions between anchors and between tag and anchor nodes. We use an experimental setup with 1 tag and 8 anchor nodes to compare the accuracy gains obtained by using both simple algorithms and more complex machine learning (ML) based algorithms applied on the channel impulse responses of anchor pairs. By selecting the best anchor combinations our algorithms can reduce the positioning error by 75\% (assuming perfectly known ground truth), by 19\% using realistically low complexity algorithms and by $38 \%$ for ML based algorithms.
\end{abstract}

Index Terms-ultra wideband (UWB), indoor localization, Time Difference of Arrival (TDoA), Machine Learning (ML), non-line-of-sight (NLOS)

\section{INTRODUCTION}

Indoor localization applications are gaining popularity in numerous application domains: ultra wideband (UWB) is used in applications for sports player tracking [1], [2], warehouse inventory taking [3], [4] and industry 4.0 autonomous navigation [5], [6]. These advancements are all supported by the recent rush to commercialization of the UWB technology. Major smartphone manufacturers incorporated the technology in their flagship phones (e.g. the iPhone 11, Galaxy Note s20) bringing the UWB localization technology to everyone's pocket.

UWB utilizes a large transmission bandwidth to provide a high resilience against multipath fading [7], a high temporal resolution and a low transmission power. This accurate timing resolution is exploited to accurately calculate positions using a variety of localization techniques such as two way ranging (TWR), active/passive time difference of arrival (TDoA) and angle of arrival (AoA). (i) TWR techniques calculate the distance between devices using the time of flight between two nodes (requiring at least two packets per distance calculation), making these techniques robust against clock drift errors and avoiding the need for synchronization between anchors. (ii) In contrast, active TDoA techniques calculate the position of the mobile tag based on only a single UWB transmission from the tag [8], [9]. In this case, the position is calculated using the difference in arrival time between different anchor pairs. The advantage of TDoA positioning is the better scalability and low energy consumption of the mobile tag, as battery powered mobile tags only need to transmit a single packet and the fixed anchors handle all other overhead, e.g., clock synchronization to compensate clock drift errors. This synchronization can be done wired or wireless. The authors of [10] present a TDoA positioning system with a mean error of $15-20 \mathrm{~cm}$ in a simple environment when the tag was placed in the middle of the room. In passive TDoA localization, only the anchor nodes are transmitting UWB pulses. The mobile tags listen to packets from the anchors and calculate their position based on these arrival times, allowing an unlimited number of tags and better privacy of their position. (iii) Finally, AoA techniques predict the angle between 2 nodes based on the arrival time of the packet at different antenna elements. AoA is less resilient to reflected signals but knowing 2 angles is sufficient to calculate the position. Due to limited hardware availability this technique is currently less widespread. Hybrid forms with combination of TWR, TDoA and AoA are also possible.

The main innovations of this paper are as follows.

- The accuracy of TDoA UWB localization systems in different scenarios (including line-of-sight (LOS) and non-line-of-sight (NLOS) conditions) is evaluated.

- Six algorithms to select a subset of anchor pairs are proposed, including simple heuristics and more complex machine learning based algorithms.

- The paper experimentally evaluates the accuracy gains as well as the complexity trade-offs for the different algorithms in different scenarios.

The remainder of this paper is structured as follows. Related work on UWB NLOS detection and anchor selection is discussed in Section II. In Section III we discuss relevant background information on the localization system, the measurement methodology and the dataset collection. The different anchor pair selection algorithms are introduced in Section IV, followed by the machine learning model in Section V. In 
Section VI, the influence of numbers of anchor pairs is investigated for the TDoA localization system. The results from the anchor pair selection algorithms are presented in Section VII. Finally, in Section VIII conclusions are formulated.

\section{RELATED WORK}

UWB line-of-sight detection. To improve the localization accuracy, a first option is to perform LOS/NLOS detection in TWR systems [11]-[13]. These techniques analyze the UWB channel impulse response to detect if a signal was received from a secondary reflection path or received directly. This allows positioning systems to classify the incoming signals and to discard unreliable NLOS ranges, thereby reducing the positioning error. Filtering out the NLOS links improved the average positioning mean error of UWB systems from 1.93 to $0.59 \mathrm{~m}$ for a setup with 3 anchor nodes in [11].

UWB error mitigation. A second approach is to estimate the error and to use correction techniques to compensate this error. In [14], the authors used machine learning techniques on the channel impulse response (CIR) to predict and correct the TWR error in an industrial environment. They achieved an increase of accuracy with $266 \%$. Similarly, the authors of [15] investigated error correction capabilities in LOS, soft NLOS and hard NLOS conditions and concluded that the mitigation of the error in LOS conditions is rather limited. However, a big improvement could be achieved in several NLOS scenarios. In contrast to the above solutions that focus on TWR, TDoA accuracy improvements are less studied. In both TDoA variants, the position is always calculated using a difference in arrival time between 2 anchors nodes, therefore making the LOS/NLOS detection algorithms and error correction approaches designed for TWR, which rely on a single range, not suitable. To the best of our knowledge, no machine learning based TDoA error correction has been proposed before.

Non-UWB based anchor selection for TDOA localization. In TDoA systems, rather than calculating the position on all receivers, measurements from a subset of anchor nodes can be discarded/corrected. Several approaches have been proposed for non-UWB technologies. In [16], researchers propose a new TDoA variant with compensation of clock offset for wireless sensor networks. Each anchor takes a turn to periodically broadcast synchronization messages to limit the influence of clock offset and clock skew. The authors of [17] investigated the number of required anchor stations for a TDoA approach. The accuracy increases with the number of anchor stations, but the increase in accuracy for adding new anchors diminishes for higher numbers of anchors. As the use of more anchors also has disadvantages, a trade-off exists between accuracy and scalability. Selecting the best anchors could improve the systems performance. Finally, selecting a subset of the sensor devices for passive localization has been proposed in [18]. In this work, it is presented that the error for the $k$-nearest sensors is higher than the error for $k$ smart selected sensor nodes.

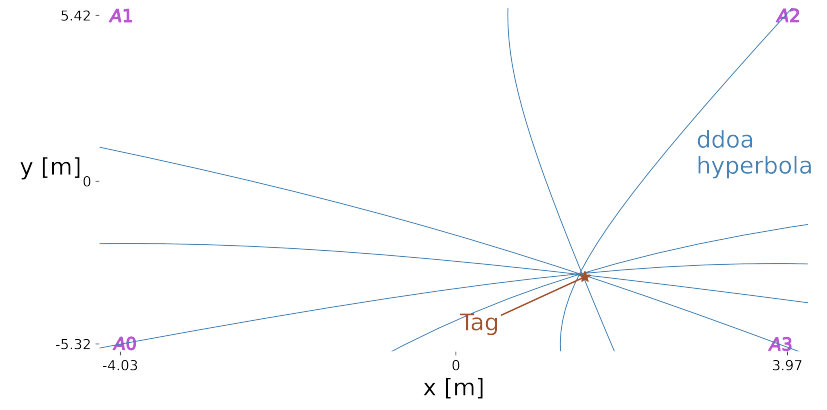

Fig. 1: Measurement setup with 4 anchor nodes and one mobile tag. All six anchor pairs result in one hyperbola, representing the difference in distance between the tag and both anchors, intersecting at the position of the tag.

\section{MEASUREMENT SETUP AND BACKGROUND}

\section{A. Time Difference of Arrival}

This paper focuses on active TDoA systems. Hereby, the anchors receive one packet, originating from the tag node. The anchor nodes mark the exact time of reception of this packet and an edge node calculates the time difference for each anchor pair (in nanoseconds), based on this difference and using the well-known propagation speed of the packets, the difference in distance between the tag and both anchors can be calculated. The two receiving timestamps therefore result in one TDoA and a corresponding distance difference of arrival (DDoA), expressing the difference in arrival in $\mathrm{mm}$. All possible tag positions with the same distance difference from 2 anchors are resembled by a hyperbola (in 2D) or hyperboloid (in 3D). If both receiving times are exactly the same, the tag will be at equal distance from both nodes and the possible tag positions are represented by a straight line/plane. Figure 1 shows the 2D hyperbolas intersecting around the mobile tag's position. The number of possible DDoAs in a cell with $n$ anchors contributing to the position can be calculated with following formula:

$$
\text { \#DDoAs }=\frac{n !}{(n-2) ! * 2 !}
$$

Assuming perfect LOS conditions, only 2 or 3 anchors are required to determine the position of the tag. As such, in this case the main accuracy of the system depends on the anchors geometric configuration and UWB timestamp quality. Complex environments with mixed LOS/NLOS conditions however also provide erroneous DDoAs, shifting the hyperbolas and introducing errors in the position approximation. The main goal of this paper is to detect and filter out the hyperbolas from these unreliable links to improve the localization accuracy.

\section{B. Wireless clock synchronization}

To compensate for clock drift errors in the anchor nodes [10], [19], the receiving times of all anchors will be translated to the time reference of the anchor 0 node. This anchor 0 node will periodically send an UWB pulse. Every other anchor that 


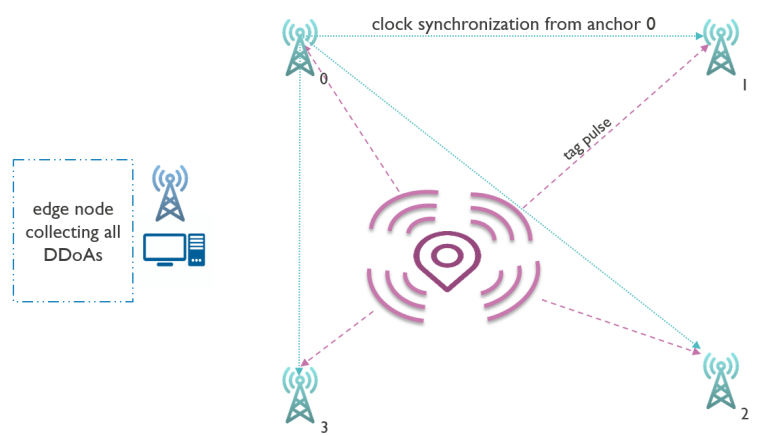

Fig. 2: System architecture with mobile tag node, fixed anchor nodes and one edge node. UWB pulses are transmitted from the tag for localization and from the anchor 0 for synchronization. The localization algorithm is at the edge node.

receives this pulse will calculate his relative clock skew $\rho$ to this anchor 0 reference time with following equation [19]:

$$
\begin{gathered}
\rho=\frac{T_{j}^{0, t x}-T_{j-1}^{0, t x}}{T_{j}^{0, r x}-T_{j-1}^{0, r x}} \\
T_{j}^{t, r x}=T_{j}^{0, r x}+\rho *\left(T_{j}^{t, r x}-T_{j-1}^{0, r x}\right)
\end{gathered}
$$

with $T_{j}^{0, t x}$ and $T_{j}^{0, r x}$ the transmitting and receiving timestamps of the synchronization UWB pulse originating from anchor 0 at time $\mathrm{j}$ and $T_{j}^{t, r x}$ the receiving timestamp of the tag's pulse at time $\mathrm{j}$.

\section{Positioning system and hardware}

The global system architecture is shown in Figure 2. The system that was deployed utilizes wireless synchronization between anchor 0 and the other anchor nodes. All DDoA data is wirelessly transmitted to a server node using sub-GHz radios, making the system represent the most generic use case whereby no cabling is required at all during the installation of the system [3]. The position of the tag is calculated at the edge server, which can send these again to the mobile node if required by its preferred communication technology. In general, the solution presented in this paper can also be applied to wired synchronization system, as well to situations in which the positions are estimated by the mobile unit itself.

The measurements are performed with 9 Wi-Pos devices [7] (Figure 3b). These devices support both UWB (DW1000) and a wireless sub-GHz (CC1200) backbone for MAC synchronization, scheduling and reporting of the timestamps. The 8 anchors are mounted at fixed positions in a cuboid setup in the IIoT-lab (Figure 3a) [20]. The open measurement space is indicated on the map in Figure 3c. All data is collected using one fixed UWB PHY setting: channel 5, a bitrate of $850 \mathrm{kpbs}$, a $64 \mathrm{MHz}$ PRF and 512 symbols of preamble.

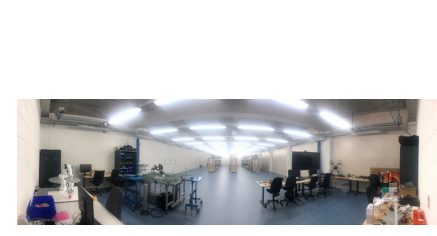

(a) The iiot lab.

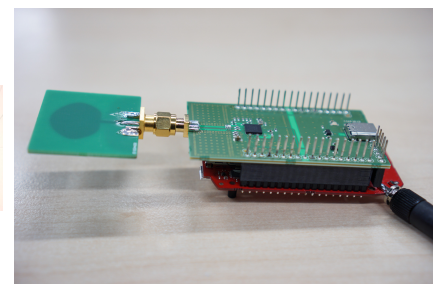

(b) Used hardware platform

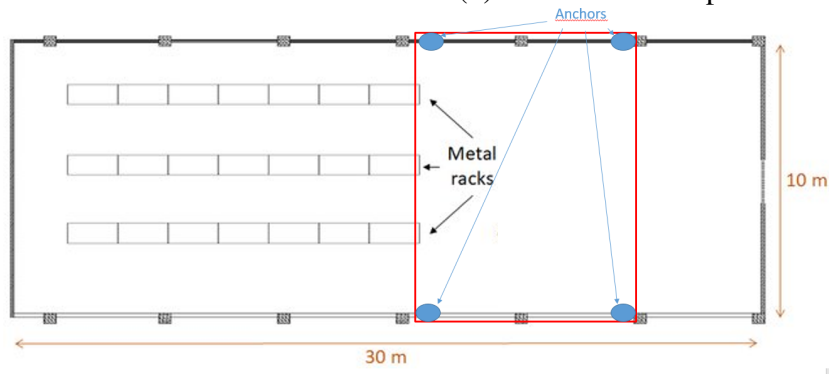

(c) Map of the lab and environment space. Accurate Mocap ground truth is available within the red square.

Fig. 3: The measurements were performed in the open space area of the iiot-lab

\section{Data collection}

Together with the collection of the UWB data, the ground truth is acquired by a Qualisys motion capturing system with mm-accuracy ground truth [21]. During the data collection, 3 different scenarios were evaluated (see Figure 4a). For the first scenario: LOS, all anchors and the tag are in LOS conditions with no obstructions. The second scenario, NLOS tag, introduces NLOS between the tag and some of the anchors. In the third scenario, NLOS tag + anchor, extra obstructions between anchor 0 and the other anchors are added. In total 6987 different positions on 62 different locations were evaluated. Outlier DDoAs with wrongly synchronized clocks were filtered from the dataset before applying any algorithm. The NLOS was introduced with moving carts, placed at random locations on the path between the nodes. The use of random locations ensures a more general conclusion for use in other environments as the algorithm doesn't know this. The behavior (attenuating and blocking of the signal) of the moving carts is similar to fixed walls in challenging environments.

\section{ANCHOR PAIR SELECTION ALGORITHMS}

Anchor pair selection is performed on the data sets using post-processing on the edge server. All algorithms are evaluated on the same dataset to limit the influence of physical differences between 2 runs. To have a generalized approach, no environment information is taken into account when selecting subsets. The different proposed approaches are discussed below and an overview of all evaluated algorithms is given in Figure 4b.

\section{A. General / All anchors algorithm}

This positioning algorithm calculates the position using a linear least-squares algorithm by equally weighting the input 


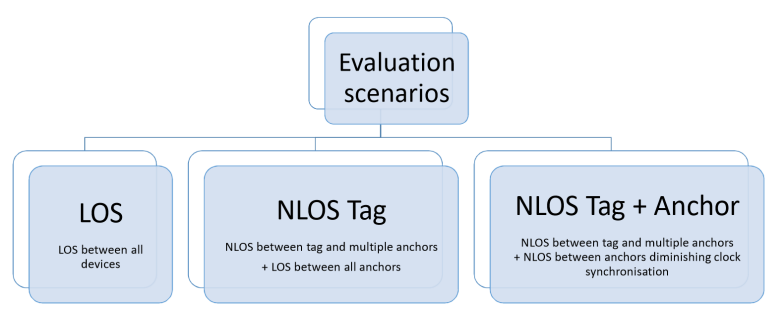

(a) Three different scenarios were evaluated.

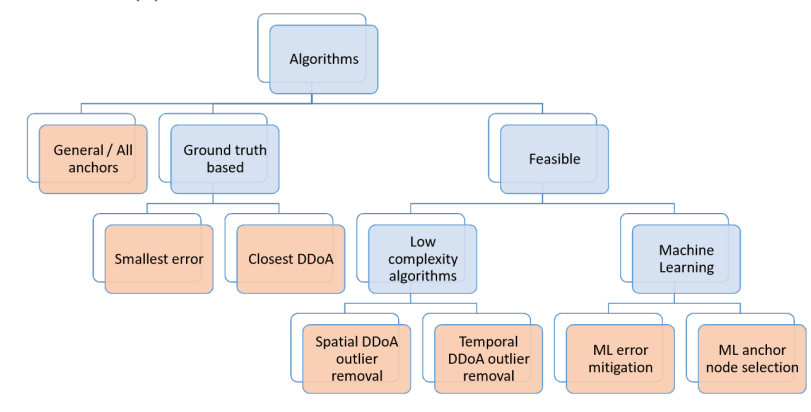

(b) Six anchor selection algorithms are proposed.

Fig. 4: Multiple algorithms are proposed to improve localization and compared in different scenarios.

from all anchors. No measurements are filtered out. Since this is the default approach in most TDoA systems, this algorithm will be used to benchmark against other algorithms.

\section{B. Ground truth based}

The first two algorithms use two sets of inputs: the available DDoA measurements and the actual ground truth position of the tag as obtained by the Motion capturing (Mocap) system. Although this ground truth information is not available in real environments, this set of algorithms provides insights on the potential improvement limits that can be gained using anchor pair selection.

1) Smallest error: With the ground truth position originating from the Mocap system and both anchor positions, it is possible to calculate the theoretical timestamp difference. This theoretical difference in timestamps is compared to the measured difference in timestamp and the introduced error, caused by physical influences on arrival times at both receivers, is ordered. The best $k$ anchors pairs, with lowest error, are selected to determine the position with a linear least square algorithm.

2) Closest DDoA: All DDoAs are ordered depending on their relative distance to the position measured with the Mocap system. The best $k$ anchors pairs are selected to determine the position. Anchor combinations with bad geometric constellation, e.g., anchors on top of each other, are therefore less likely to be included in the subset in this algorithm.

\section{Low complexity algorithms}

The next set of algorithms do not rely on ground truth information and require only the values of the DDoAs to be available at the edge node for position estimation.
1) Spatial DDoA outlier removal: First, all DDoAs are collected on the edge device and used to calculate a likely position 1 using a linear least-square algorithm (similar to the all anchors algorithm). Next, the algorithm assumes that there exist a minority of outlier DDoAs that originate from NLOS conditions and negatively impact the position estimation. To detect and remove these, all DDoAss are sorted according to their relative distance to position 1 . Finally, the closest $k$ DDoAss are assumed to be from LOS conditions, and the least-square algorithm is run a second time using only these $k$ DDoAss resulting in the final position estimate.

2) Temporal DDoA outlier removal: In harsh environments, NLOS will typically have less stable DDoA distributions over time. This time based selection algorithm will take into account the recent history of the previous DDoAs from all anchor pairs and select the $k$ anchors pairs with the smallest standard deviation. The time window for comparing with previous samples is set to be very short to limit the influence of fast moving tags on the DDoA standard deviation.

\section{Machine Learning}

Albeit more computationally complex to implement on embedded devices, machine learning (ML) algorithms accuracy improvements have been applied to the channel impulse responses obtained from UWB TWR systems to reduce the positioning error. However, in TDoA environments a DDoA is dependent on the channel impulse responses from 2 anchors, hence requiring a different approach. We consider two machine learning-based approaches, which are described more in details in the next Section.

1) ML error mitigation: Machine learning is used to predict DDoA errors and to correct the individual DDoAs based on the CIR input from both anchors. These corrected DDoAs will be used to calculate a position based on all anchor pairs.

2) ML anchor pair selection: In this algorithm, machine learning is also used to predict the error. However, rather than correcting the error, in case sufficient high-quality DDoAs are present only the best $k$ DDoAs are selected to calculate the position.

An overview of the different algorithms is given in Table I. The ground truth based algorithms are considered non-feasible as they require a more accurate position source for their decision making. They are included to illustrate the maximum obtainable impact of anchor pair selection. Machine learning approaches require CIR data which has to be transmitted to the edge server, thus requiring a high-throughput backbone. The computation itself will introduce a small extra latency, but can be done on e.g., a resource-constrained edge node [14]. Table I also discusses the suitability of the algorithms for different use cases. Non-moving targets are handled well by all algorithms. Some algorithms are designed to estimate one position using prior information and are hence less suited for fast moving objects. Finally, a complexity analysis of each algorithm is added. By selecting a subset of $k$ anchor pairs out of the $n$ ! combinations with $n$ anchors, the complexity of the localization calculations are reduced drastically. 


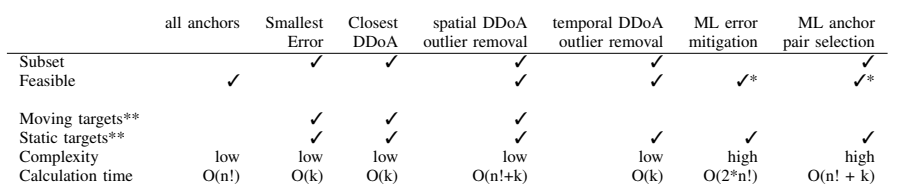

TABLE I: High level parameters of the algorithms. The complexity of the algorithms is shown as a function of the number of available anchors $n$ and subset size $k$.*if high throughput links from anchor to edge server are present. **assumed in challenging NLOS environment.

\section{MACHINE LEARNING MODEL}

To employ a data-driven machine learning model, we have implemented a convolutional neural network (CNN) architecture, which has shown great success in previous works related to TWR error correction [14]. The input of the neural network consists of two CIRs for each anchor pair. We have pre-processed each CIR in such a way to only focus on relevant samples concerning direct path and reflected paths of the UWB signals. These CIRs contain information about the environment and potential NLOS signal characteristics, which provides the ML model the possibility to discover errors made by the on chip leading-edge algorithm. An overview of this pre-processing step is provided in Figure 5. First, for each CIR the first path is gathered and used to center the CIR. With this CIR alignment we try to induce the first path index as a feature, so the CNN knows where the leading-edge algorithm positioned this first path, as this is not always logged in the same position. Additionally, we keep only 80 samples, 20 before the first path and 60 after. This allows the CNN to learn the noise floor before the first path and multipath components after. The architecture of the CNN is shown in Figure 6. The model consists of 4 convolutional layers with $32,16,8$ and 4 filters respectively. The first three are 1D to convolute only in the time dimension, while the last one convolutes over the second dimension as well to extract features from the combined anchor CIRs. Following the convolutional layers, there are 4 dense fully connected (FC) layers and 1 output layer, with one neuron. This last neuron outputs the predicted error. Furthermore, we have experimentally determined that adding batch-normalization after the first convolutional layers and before the FC layers improve accuracy, as well as adding dropout of $25 \%$ and 12 regularizes in the convolutional layers. The model is trained in a supervised fashion by traditionally updating the weights using back-propagation. More specifically, the weights are optimized using Adams gradient descent algorithm which minimizes a mean absolute error loss function with a batch size of 256 .

\section{OPTIMAL NUMBER OF ANCHOR PAIRS}

First, we study how the number of anchor pairs impacts the overall accuracy. The number of anchor pairs and their corresponding error is shown in Figure 7 for the smallest error algorithm in all the different scenarios. Initially, for small numbers of considered anchor pairs, the accuracy of the TDoA system increases significantly by adding more.

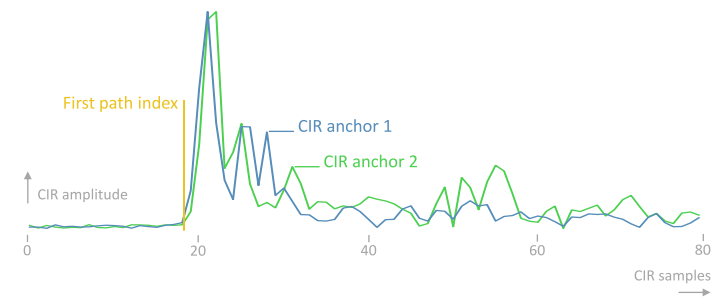

Fig. 5: The model uses the channel impulse responses from 2 anchors to determine the error between for that anchor pair.

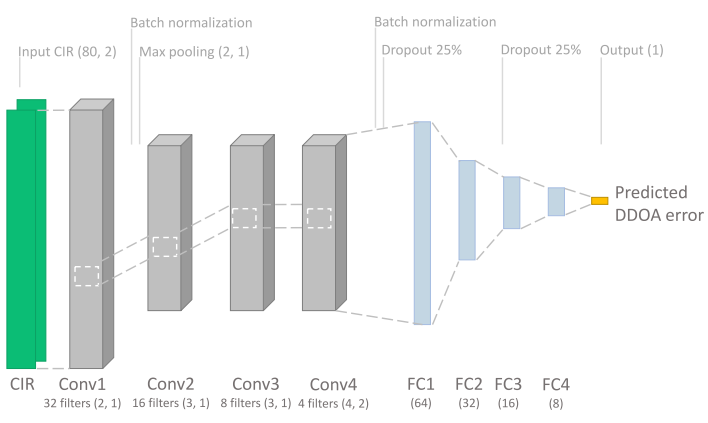

Fig. 6: The CNN model for DDoA classification

After including 8 anchor pairs, the accuracy is actually slightly increasing in the LOS scenario and the NLOS tag scenario up to the level of the general algorithm for the temporal DDoA outlier removal algorithm. Reducing the number of anchor pairs further has a minor influence on the accuracy, with a higher gain in complexity and speed for calculating the final position. For the NLOS tag + anchor scenario, the error is minimal for smaller subsets and adding extra (erroneous) anchor pairs actually increases the error.

\section{RESULTS}

Now that we know the impact of reducing the number of anchor pairs, this section analyses which algorithms are best suited for the actual anchor pair selection. To compare the algorithms we utilize three evaluation criteria: mean absolute error (MAE), 75th percentile error and 95th percentile error. In case many outliers are present, the MAE could be relatively high. In these cases the 75th percentile is useful to consider as well as mostly the highest errors influence the performance of the system. The cumulative distribution function (CDF) of the different algorithms can be found in Figure 8a and detailed outcomes are shown in Table II.

\section{A. Scenario 1: $L O S$}

For this first scenario, we measured 6 locations in the open space environment (Figure 3c). In total, 961 transmitted UWB pulses originating from the tag are collected for this scenario.

1) General / All anchors algorithm: The general algorithm was able to determine the tag's position with a 2-dimensional mean error of $97 \mathrm{~mm}$ with a 75th and 95th percentile of 119 and $153 \mathrm{~mm}$ respectively. 


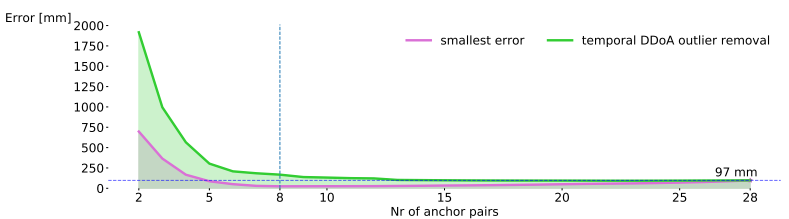

(a) Scenario 1: LOS

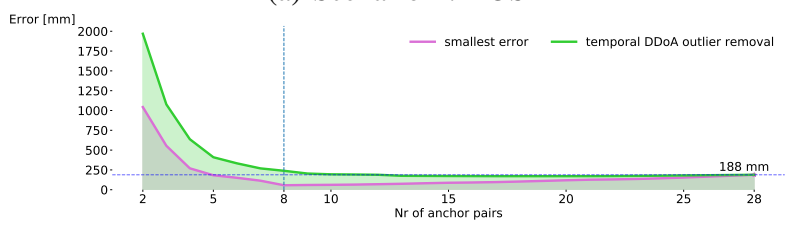

(b) Scenario 2: NLOS tag

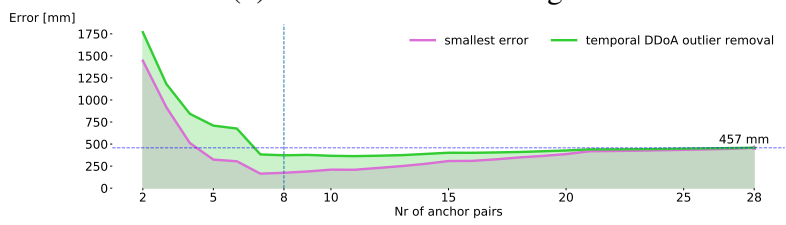

(c) Scenario 3: NLOS tag + anchor

Fig. 7: The mean error initially decreases when adding anchor pairs for both demonstrated algorithms, but the accuracy gains for adding additional anchor pairs shows diminishing returns. Selecting a good subset might be recommended in all scenarios.

2) Ground truth based algorithms: The smallest error algorithm has a mean error of $24 \mathrm{~mm}$ with and 75 and 95 th percentiles at $29 \mathrm{~mm}$ and $64 \mathrm{~mm}$. This algorithm is on average $75 \%$ better than the typically used all anchor algorithm, demonstrating the importance of correct anchor pair selection even in LOS conditions. The closest DDoA algorithm with positions based on 8 DDoAs has a MAE, 75 and 95th percentile of 89, 119 and $167 \mathrm{~mm}$ respectively. This algorithm does improve the mean positioning error but with the negative side that it increases the higher percentile compared to the general algorithm.

3) Low complexity algorithms: The spatial DDoA outlier removal algorithmic approach, increases mean $(121 \mathrm{~mm}), 75$ th $(154 \mathrm{~mm})$ and 95th $(199 \mathrm{~mm})$ percentile. The second low complexity algorithm is the temporal DDoA outlier removal algorithm. The mean error increases significantly to $167 \mathrm{~mm}$ $(+70 \mathrm{~mm})$. The mean absolute error is close to the $166 \mathrm{~mm}$ of the 75th percentile, showing that some far outliers influence the results. As such, both algorithms negatively influence the accuracy in LOS conditions.

4) Machine Learning: In the LOS scenario, the accuracy improves after applying the ML error mitigation. The mean positioning error is now only $61 \mathrm{~mm}$, improving the accuracy by $38 \% .75 \%$ of the points are within the $83 \mathrm{~mm}$ error range and $95 \%$ of the errors are smaller than $115 \mathrm{~mm}$. Selecting the 8 DDoAs with the ML anchor pair selection algorithm has relatively little impact on the mean accuracy in LOS conditions, resulting in a mean absolute error of $97 \mathrm{~mm}$ but it has higher percentiles of 126 and $192 \mathrm{~mm}$.

\section{B. Scenario 2: NLOS tag}

In this second scenario, we included 33 locations in the lab to have a more general dataset for taking conclusions as the environment (location of the obstructions) also has an influence. The CDF overview is given in Figure 8b.

1) General algorithm: For the general positioning method, this scenario is already more difficult. By adding NLOS in the scenario, the mean absolute error has increased by 91 $\mathrm{mm}$ compared to the LOS scenario to $188 \mathrm{~mm}$. The 75 and 95th percentiles are significantly higher at $262 \mathrm{~mm}$ and 424 $\mathrm{mm}$, respectively. The algorithm is suffering from less accurate DDoA measurements.

2) Ground truth based algorithms: In this more challenging scenario, the smallest error algorithm has a mean error of $57 \mathrm{~mm}$ and low percentiles (66 and $187 \mathrm{~mm}$ ). As such, the improvements of this algorithm remain stable around 70\%. The closest DDoA algorithm now also improves the performance. The mean error is now $133 \mathrm{~mm}$, the 75thpercentile $182 \mathrm{~mm}$ and the 95th is $289 \mathrm{~mm}$, an improvement of around $31 \%$.

3) Low complexity algorithms: The low complexity algorithms don't positively impact the accuracy in this scenario when compared to the general all anchor node algorithm. For the spatial DDoA outlier removal algorithm the mean is slightly higher at $203 \mathrm{~mm}$. The algorithm locates $75 \%$ of the points within $270 \mathrm{~mm}$ of the true position and $95 \%$ have errors less then $422 \mathrm{~mm}$, a small improvement compared to the general algorithm. For the temporal DDoA outlier removal algorithm, the mean value is $239 \mathrm{~mm}$ with high percentiles as well (255 and $436 \mathrm{~mm}$ ).

4) Machine Learning: For the machine learning approach, the ML error mitigation again has a positive impact, lowering the mean absolute error to $134 \mathrm{~mm}$, a decrease with $27 \%$ compared to localization without correction. $75 \%$ of the positions are within $176 \mathrm{~mm}$ and $95 \%$ not farther than $330 \mathrm{~mm}$ of the real position. In contrast, the performance of the (ML anchor pair selection) is much worse, actually increasing the mean error to $327 \mathrm{~mm}$.

\section{Scenario 3: NLOS tag + anchor}

The third scenario is the most difficult one since there are NLOS errors introduced in the calculation of the clock drift of different anchors and by obstruction of the path between tag and anchor. The cumulative distribution functions can be found in Figure 8c. Due to its high complexity, the majority of the evaluated algorithms significantly improve the accuracy in this scenario compared to the all anchor node algorithm.

1) General algorithm: Using the standard all anchor node approach we obtain a very high mean error of $457 \mathrm{~mm}$. Information from the 75th percentile $(524 \mathrm{~mm})$ and 95th percentile $(1230 \mathrm{~mm})$ shows that this high mean error could be explained by outlier positions with high errors. The other algorithms will mostly work on filtering the best DDoAs and improving of this position. 


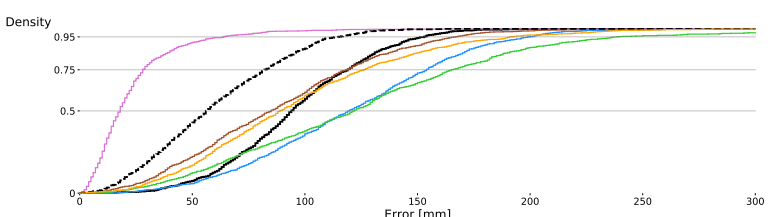

(a) CDF scenario 1: LOS

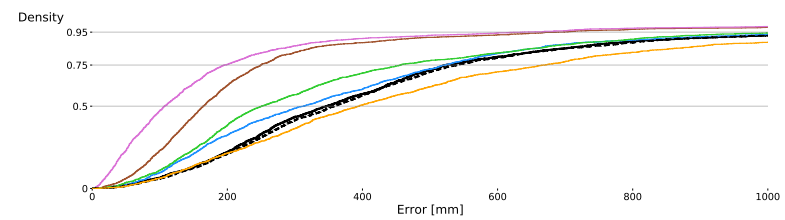

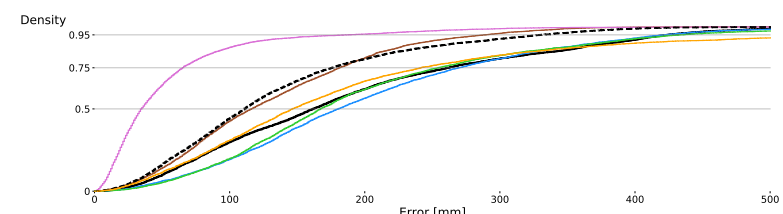

(b) CDF scenario 2: NLOS tag

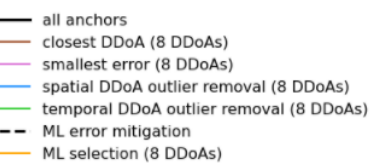

(c) CDF scenario 3: NLOS tag + anchor

Fig. 8: Cumulative distribution functions showing the positioning error in the three scenarios. Several algorithms improve the accuracy in all scenarios (e.g. smallest error, closest ddoa), whereas other algorithms (such as the low complexity algorithms) positively impact the error in one or two scenarios while negatively impacting the error in other scenarios.

\begin{tabular}{|c|c|c|c|c|c|c|c|c|}
\hline \multirow[b]{2}{*}{ number DDoAs } & & General & $\begin{array}{r}\text { Smallest } \\
\text { Error }\end{array}$ & $\begin{array}{l}\text { Closest } \\
\text { DDoA }\end{array}$ & $\begin{array}{l}\text { spatial DDoA } \\
\text { outlier removal }\end{array}$ & $\begin{array}{l}\text { temporal DDoA } \\
\text { outlier removal }\end{array}$ & $\begin{array}{l}\text { ML error } \\
\text { mitigation }\end{array}$ & $\begin{array}{r}\text { ML anchor } \\
\text { pair selection }\end{array}$ \\
\hline & & 28 & 8 & 8 & 8 & 8 & 28 & 8 \\
\hline \multirow{3}{*}{ LOS } & mean & $97 \mathrm{~mm}$ & $-75 \%$ & $-8 \%$ & $25 \%$ & $73 \%$ & $-38 \%$ & $0 \%$ \\
\hline & 75th & $119 \mathrm{~mm}$ & $-76 \%$ & $0 \%$ & $30 \%$ & $39 \%$ & $-31 \%$ & $6 \%$ \\
\hline & 95th & $153 \mathrm{~mm}$ & $-58 \%$ & $9 \%$ & $30 \%$ & $56 \%$ & $-25 \%$ & $25 \%$ \\
\hline \multirow{4}{*}{ NLOS tag } & mean & $188 \mathrm{~mm}$ & $-70 \%$ & $-30 \%$ & $8 \%$ & $27 \%$ & $-29 \%$ & $74 \%$ \\
\hline & 75th & $262 \mathrm{~mm}$ & $-75 \%$ & $-31 \%$ & $3 \%$ & $-3 \%$ & $-33 \%$ & $-8 \%$ \\
\hline & 95th & $424 \mathrm{~mm}$ & $-56 \%$ & $-32 \%$ & $0 \%$ & $3 \%$ & $-22 \%$ & $110 \%$ \\
\hline & mean & $457 \mathrm{~mm}$ & $-62 \%$ & $-51 \%$ & $-9 \%$ & $-19 \%$ & $2 \%$ & $16 \%$ \\
\hline \multirow[t]{2}{*}{ NLOS tag + anchor } & 75th & $524 \mathrm{~mm}$ & $-63 \%$ & $-52 \%$ & $-0 \%$ & $-13 \%$ & $3 \%$ & $28 \%$ \\
\hline & 95th & $1230 \mathrm{~mm}$ & $-44 \%$ & $-48 \%$ & $-9 \%$ & $-16 \%$ & $-1 \%$ & $10 \%$ \\
\hline
\end{tabular}

TABLE II: The mean and percentile error improvements of the anchor pair selection algorithms for the different scenarios. Among the feasible implementations, the machine learning based approaches outperform the low complexity algorithms in the LOS and NLOS tag scenarios, meanwhile the low complexity algorithms perform better for the NLOS tag + anchor scenario.

2) Ground truth based algorithms: Similar to the results for the other two scenarios, we also find an improvement of the position with the smallest error algorithm. The mean error is reduced to $174 \mathrm{~mm}$ and the percentiles to $196 \mathrm{~mm}$ (75th) and $636 \mathrm{~mm}$ (95th). For this scenario, the closest DDoA algorithm also succeeds in lowering the mean error $(226 \mathrm{~mm})$ to a level below both percentiles (250 and $689 \mathrm{~mm}$ ). The gains for selecting a subset of 8 DDoAs for the least squares algorithm for position approximation are again significant.

3) Low complexity algorithms: The low complexity algorithms significantly improve the robustness of the system. For the spatial DDoA outlier removal algorithm, the mean error is now $419 \mathrm{~mm}$ (-9\% compared to all anchors). The gains are lower for the 75th percentile $(-0 \%, 523 \mathrm{~mm})$. For the 95th percentile $9 \%$ reduction in error could be observed. This 95 th percentile error is at $1117 \mathrm{~mm}$. Also, the temporal DDoA outlier removal algorithm result in improvement with a MAE of $373 \mathrm{~mm} \mathrm{(-19 \% )}$ and 458 and $1039 \mathrm{~mm}$ for both percentiles.

4) Machine Learning: The machine learning algorithms are trained and applied on the CIRs from the tag's pulse. By adding NLOS between the anchors in the same, we expect difficulties for the machine learning to predict the error in these cases. By just mitigating the error in the ML error mitigation algorithm, the MAE increases with 2 percent to
$466 \mathrm{~mm}$ compared to the general algorithm. The percentiles are similar to the general scenario at 540 and $1219 \mathrm{~mm}$. Although these are significant improvements in the other scenarios, this algorithm isn't improving positioning. By selecting a subset of the ranges in the ML anchor pair selection, the mean error increases to $531 \mathrm{~mm}$, showing that this approach is also not beneficial in very complex environments. The percentiles are following the same trend as the other scenarios (670 mm (75th) and $1356 \mathrm{~mm}$ (95th)).

By considering 3 different scenarios for the algorithms, some clear conclusions are rising. For all three scenarios, the smallest error algorithm outperforms the other algorithms and in particular the general algorithm. Smartly selecting a subset of anchor pairs is therefore recommended for positioning of TDoA tags. Although, this scenario is ground truth based and not feasible, the other algorithms improve accuracy in specific scenarios. The ML error mitigation model improves TDoA localization in all scenarios where clock synchronization can be guaranteed, while the low complexity algorithms are covering this scenario completely and improving the robustness there. Depending on the use case, the UWB system designer could make a thorough decision on optimization of the TDoA localization algorithm. 


\section{CONCLUSIONS}

The mean absolute positioning error in time difference of arrival UWB localization systems increases when a significant number of NLOS links are present between mobile tag and anchor nodes. This error can be lowered by selecting the best subset of anchor pairs. Although anchor selection has shown to reduce the localization error in TWR UWB systems, solutions for error reduction in TDoA systems were still lacking. For this anchor selection 6 new algorithms were evaluated in 3 different scenarios.

Two ground truth based algorithms for selecting $k$ anchor pairs were investigated as a benchmark opportunity into the maximum obtainable error correction gains when using anchor pair selection. By optimally selecting the 8 best anchor pairs to use, we found that the MAE can be reduced up to $-75 \%$ in LOS conditions and $-70 \%$ in NLOS conditions, demonstrating the importance of good anchor pair selection algorithms.

Two simple heuristic algorithms have been proposed that improved the accuracy in complex scenarios where both anchors and tag suffer from NLOS links. Especially when anchors have wireless synchronization problems due to blocked channels between the anchor nodes, the algorithms succeed in lowering the mean absolute error with $9 \%$ and $19 \%$ for these 2 algorithms respectively. However, this comes at a cost of decreased accuracy in LOS conditions. As such, these algorithms should be used when wireless synchronization proves difficult (e.g. due to obstacles) or when this situation can easily be detected.

A final approach relies on applying ML on the channel impulse responses of both anchors to try to predict the error of the DDoA. This machine learning based error correction improves the accuracy in two of the three considered scenarios: a mean error reduction of $38 \%$ in the LOS scenario, $29 \%$ in the NLOS tag scenario. In the third scenario, the biggest errors were introduced during the clock synchronization due to NLOS effects. As such, this algorithm is beneficial to apply in well synchronized conditions, but comes at the cost of larger data throughput and complexity to collect CIR values.

\section{ACKNOWLEDGMENT}

FWO EOS PROJECT 30452698 - MUSE-WINET (MUltiSErvice WIreless NETwork)

\section{REFERENCES}

[1] M. Ridolfi, S. Vandermeeren, J. Defraye, H. Steendam, J. Gerlo, D. De Clercq, J. Hoebeke, and E. De Poorter, "Experimental evaluation of uwb indoor positioning for sport postures," Sensors, vol. 18, no. 1, 2018. [Online]. Available: https://www.mdpi.com/1424-8220/18/1/168

[2] A. Bastida-Castillo, C. D. Gómez-Carmona, E. De la Cruz-Sánchez, X. Reche-Royo, S. J. Ibáñez, and J. Pino Ortega, "Accuracy and inter-unit reliability of ultra-wide-band tracking system in indoor exercise," Applied Sciences, vol. 9, no. 5, 2019. [Online]. Available: https://www.mdpi.com/2076-3417/9/5/939

[3] N. Macoir, J. Bauwens, B. Jooris, B. Van Herbruggen, J. Rossey, J. Hoebeke, and E. De Poorter, "Uwb localization with batterypowered wireless backbone for drone-based inventory management," SENSORS, vol. 19, no. 3, pp. 467:1-467:18, 2019. [Online]. Available: http://dx.doi.org/10.3390/s19030467
[4] Z. Wang, S. Li, Z. Zhang, F. Lv, and Y. Hou, "Research on uwb positioning accuracy in warehouse environment," Procedia Computer Science, vol. 131, pp. 946 - 951, 2018, recent Advancement in Information and Communication Technology:. [Online]. Available: http://www.sciencedirect.com/science/article/pii/S1877050918306112

[5] J. Tiemann, F. Schweikowski, and C. Wietfeld, "Design of an uwb indoor-positioning system for uav navigation in gnss-denied environments," in 2015 International Conference on Indoor Positioning and Indoor Navigation (IPIN), 2015, pp. 1-7.

[6] M. Ridolfi, N. Macoir, J. Vanhie-Van Gerwen, J. Rossey, J. Hoebeke, and E. De Poorter, "Testbed for warehouse automation experiments using mobile agvs and drones," in IEEE CONFERENCE ON COMPUTER COMMUNICATIONS WORKSHOPS (IEEE INFOCOM 2019 WKSHPS). IEEE, 2019, pp. 919-920. [Online]. Available: http://dx.doi.org/10.1109/infcomw.2019.8845218

[7] B. Van Herbruggen, B. Jooris, J. Rossey, M. Ridolfi, N. Macoir, Q. Van den Brande, S. Lemey, and E. De Poorter, "Wi-pos: A low-cost, open source ultra-wideband (uwb) hardware platform with long range sub-ghz backbone," Sensors, vol. 19, no. 7, 2019. [Online]. Available: https://www.mdpi.com/1424-8220/19/7/1548

[8] R. Ye and H. Liu, "Uwb tdoa localization system: Receiver configuration analysis," in 2010 International Symposium on Signals, Systems and Electronics, vol. 1, 2010, pp. 1-4.

[9] B. Choi, K. La, and S. Lee, "Uwb tdoa/toa measurement system with wireless time synchronization and simultaneous tag and anchor positioning," in 2018 IEEE International Conference on Computational Intelligence and Virtual Environments for Measurement Systems and Applications (CIVEMSA), 2018, pp. 1-6.

[10] V. Djaja-Josko and J. Kolakowski, "A new method for wireless synchronization and tdoa error reduction in uwb positioning system," in 2016 21st International Conference on Microwave, Radar and Wireless Communications (MIKON), 2016, pp. 1-4.

[11] K. Bregar, A. Hrovat, and M. Mohorcic, "Nlos channel detection with multilayer perceptron in low-rate personal area networks for indoor localization accuracy improvement," 052016.

[12] V. Barral, C. J. Escudero, J. A. García-Naya, and P. Suárez-Casal, "Environmental cross-validation of nlos machine learning classification/mitigation with low-cost uwb positioning systems," Sensors, vol. 19, no. 24, 2019. [Online]. Available: https://www.mdpi.com/1424-8220/19/24/5438

[13] J. Kulmer, S. Hinteregger, B. Großwindhager, M. Rath, M. S. Bakr, E. Leitinger, and K. Witrisal, "Using decawave uwb transceivers for high-accuracy multipath-assisted indoor positioning," in 2017 IEEE International Conference on Communications Workshops (ICC Workshops), 2017, pp. 1239-1245.

[14] J. Fontaine, M. Ridolfi, B. Van Herbruggen, A. Shahid, and E. De Poorter, "Edge inference for uwb ranging error correction using autoencoders," IEEE Access, vol. 8, pp. $139143-139$ 155, 2020.

[15] V. Barral, C. J. Escudero, J. A. García-Naya, and R. ManeiroCatoira, "Nlos identification and mitigation using low-cost uwb devices," Sensors, vol. 19, no. 16, 2019. [Online]. Available: https://www.mdpi.com/1424-8220/19/16/3464

[16] H. Xiong, Z. Chen, B. Yang, and R. Ni, "Tdoa localization algorithm with compensation of clock offset for wireless sensor networks," China Communications, vol. 12, no. 10, pp. 193-201, 2015.

[17] Z. Dai, G. Wang, X. Jin, and X. Lou, "Nearly optimal sensor selection for tdoa-based source localization in wireless sensor networks," IEEE Transactions on Vehicular Technology, vol. 69, no. 10, pp. 12031$12042,2020$.

[18] Y. Zhao, Z. Li, B. Hao, P. Wan, and L. Wang, "How to select the best sensors for tdoa and tdoa/aoa localization?" China Communications, vol. 16, no. 2, pp. 134-145, Feb 2019.

[19] A. Ledergerber, M. Hamer, and R. D'Andrea, "A robot selflocalization system using one-way ultra-wideband communication," in 2015 IEEE/RSJ International Conference on Intelligent Robots and Systems (IROS), 2015, pp. 3131-3137.

[20] IDLab. Industrial iot lab. [Online]. Available: https://www.ugent.be/ ea/idlab/en/research/research-infrastructure/industrial-iot-lab.html

[21] A. Qualisys, "Qualisys motion capture systems," URL: http://www. qualisys.com/, accessed on, pp. 09-04, 2020. 